\title{
Effects of non-invasive ventilation in subjects undergoing cardiac surgery on length of hospital stay and cardiac-pulmonary complications: a systematic review and meta-analysis
}

\author{
Qinhan Wu", Guiling Xiang", Jieqiong Song", Liang Xie, Xu Wu, Shengyu Hao, Xiaodan Wu, Zilong Liu, \\ Shanqun Li \\ Department of Pulmonary Medicine, Zhongshan Hospital, Fudan University, Shanghai 200032, China \\ Contributions: (I) Conception and design: Q Wu, S Li; (II) Administrative support: Z Liu, S Li; (III) Provision of study materials or patients: Q Wu, J \\ Song, L Xie, X Wu, S Hao, X Wu, Z Liu, S Li; (IV) Collection and assembly of data: Q Wu, G Xiang, J Song; (V) Data analysis and interpretation: \\ Q Wu; (VI) Manuscript writing: All authors; (VII) Final approval of manuscript: All authors. \\ \#These authors contributed equally to this work. \\ Correspondence to: Shanqun Li; Zilong Liu. Department of Pulmonary Medicine, Zhongshan Hospital, Fudan University, 180 Fenglin Rd., Shanghai \\ 200032, China. Email: 1sq18616880856@163.com; yjbbdx1988@126.com.
}

\begin{abstract}
Background: Cardiac surgery often leads to pulmonary complications. Non-invasive ventilation (NIV) is a mechanical ventilation modality that may help to prevent the pulmonary complications, and the role of the prophylactic use of NIV in patients after cardiac surgery remains controversial.

Methods: We searched PubMed, Embase, Web of Science and Cochrane Central for randomized controlled trials comparing the use of NIV (continues positive airway pressure or bi-level positive airway pressure) with standard treatment in post-cardiac surgery subjects without language restriction. Two investigators screened the eligible studies up to July, 2019. Meta-analysis using random effect model or fixed effect model was conducted for pulmonary complications, mortality, rate of reintubation and cardiac complications, and mean difference (MD) or standard mean difference for length of hospital stay and length of ICU stay.

Results: We included nine randomized controlled trails with 830 subjects. The use of NIV failed to reduce the risk of pulmonary complications, including atelectasis [risk rate (RR) 0.60 ; $95 \%$ confidence interval (CI): 0.28 to $1.28, \mathrm{P}=0.19$ ] and pneumonia (RR $0.27 ; 95 \% \mathrm{CI}: 0.05$ to $1.64, \mathrm{P}=0.16$ ). However, it has shortened the length of ICU stay (MD -1.00 h, $95 \%$ CI: -1.38 to $-0.63, \mathrm{P}<0.00001)$ and the length of hospital stay (MD $-1.00 \mathrm{~d}, 95 \% \mathrm{CI}:-1.12$ to $-0.87, \mathrm{P}<0.00001)$. NIV also failed to reduce the rate of reintubation (RR 0.68; $95 \%$ CI: 0.21 to $2.26, \mathrm{P}=0.53$ ) or the risk of cardiac complications (RR 0.81; 95\% CI: 0.59 to $1.13, \mathrm{P}=0.22$ ).

Conclusions: The prophylactic use of NIV immediately in post-cardiac subjects who underwent cardiac surgery might be able to shorten the length of hospital stay and the length of ICU stay, but it has no significant effect on pulmonary complications, rate of reintubation or cardiac complications.
\end{abstract}

Keywords: Cardiac surgical procedure; noninvasive ventilations; continuous positive airway pressure (CPAP); postoperative complications; length of stay; pulmonary atelectasis

Submitted Sep 14, 2019. Accepted for publication Jan 07, 2020.

doi: $10.21037 /$ jtd.2020.02.30

View this article at: http://dx.doi.org/10.21037/jtd.2020.02.30 


\section{Introduction}

According to a recent study, approximately 312.9 million major surgical procedures requiring general or spinalepidural anesthesia are performed worldwide each year (1). Postoperative pulmonary complications, which are generally defined as any pulmonary abnormality occurring in the postoperative period, usually lead to an increase in hospital length of stay, morbidity, mortality $(2,3)$, and extra costs for the health care system (4). It has been reported that 5 to 10 percent of surgical patients have reported at least one pulmonary complication (2), of which atelectasis is the most common (5). Cardiac surgery has a greater risk of developing pulmonary complications (2). A recent study reported that basilar atelectasis was detected in up to $94 \%$ of patients within 48 hours after coronary artery bypass grafting (CABG) (6). Common strategies for preventing post-cardiac operative pulmonary complications include appropriate analgesia, physiotherapy, oxygen therapy, early mobilization and ambulation. However, those strategies are not enough to reduce the incidence of the complications $(2,7,8)$.

Non-invasive ventilation (NIV) is a mechanical ventilation modality that uses a non-invasive airway interface instead of an invasive artificial airway. It has primarily been applied in patients with exacerbations of chronic obstructive pulmonary disease, cardiogenic pulmonary edema and hypoxemic respiratory failure (9).

The mechanism by which NIV exerts its effects is to increase intrathoracic pressure. It prevents alveolar collapse, which may increase functional residual capacity and arterial oxygenation, reduces respiratory workload and cardiac preload (10-13). Therefore, it may help to prevent the pulmonary complications and reduce the rate of reintubation in post-cardiac surgery subjects.

Although random controlled trials have been taken out in recent years, the role of NIV in patients after cardiac surgery remains controversial. Therefore, this meta-analysis was taken out to determine whether the prophylactic use of NIV able to reduce the incidence of adverse events, such as pulmonary complications and shorten the length of ICU and hospital stay in post-cardiac surgery subjects.

\section{Methods}

\section{Search strategy}

We developed a computerized search through PubMed, Embase (via Ovid), Web of Science and Cochrane Central, with a combination of Medical Subject Headings and free-text terms. The search included the available articles published from the establishment of the database to July, 2019. No language restrictions were imposed.

Search terms included: ("Noninvasive Ventilations" OR NIV OR NIPPV OR "Continuous Positive Airway Pressure" OR "Bilevel Continuous Positive Airway Pressure" OR CPAP OR BiPAP) AND ("Cardiac Surgical Procedures" OR "Heart Surgical Procedures" OR "Cardiac Surgery" OR "Heart Surgery" OR "Valve Replacement" OR "Coronary Artery Bypass" OR CABG) AND ("clinical trial” OR RCT OR "randomized controlled trial” OR "randomized controlled clinical trial").

\section{Study selection}

Two investigators independently screened titles and abstracts of the articles identified by the computerized search according to the inclusion criteria, and any discrepancies were resolved by the way of consensus. Based on this first assessment, we obtained the full text of all potentially relevant articles.

The inclusive criteria were: (I) subjects aged above 18 years of both genders, who underwent any type of cardiac surgery with cardiopulmonary bypass; (II) NIV was initiated in the immediate postoperative period (defined as the first 24 hours after the surgery) with continuous positive airway pressure (CPAP) mode or bi-level positive airway pressure mode (BiPAP) applied through a nasal or face mask; (III) applied one or a combination of the following interventions as control: oxygen therapy; chest physiotherapy (CP) techniques for removing secretions; breathing exercises; mobilization; incentive spirometry (IS); no intervention; (IV) reported at least one of the following outcomes: pulmonary complications (atelectasis and pneumonia), length of ICU stay, length of hospital stay, rate of reintubation, cardiac complications, mortality, 6-minute walking distance (6MWD); (V) randomized controlled trials (RCTs) with no restriction regards to intervention and follow-up length.

And we excluded studies if they fit the following description: (I) included subjects in the post operation period of other types of surgery, and subjects who went through heart transplant surgery; (II) applied NIV curatively (applied NIV only after the occurrence of adverse events); (III) applied NIV to early extubation participants.

\section{Data extraction and risk of bias assessment}

The data extraction was performed by two investigators 


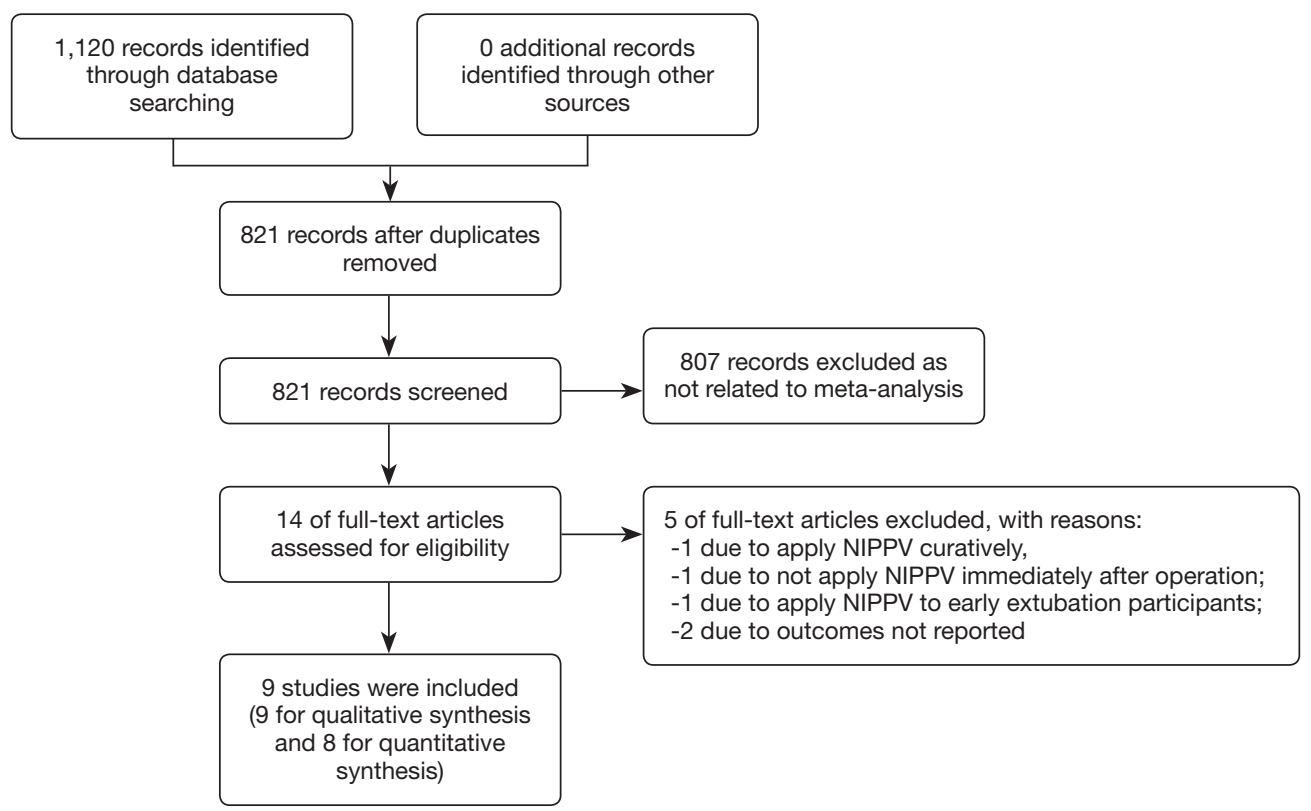

Figure 1 The study selection flow chart.

independently, any disagreements were resolved by a third investigator. The extracted information included the following: (I) the details of publication: the first author's last name, year of publication, design and location; (II) characteristics of participants: the sample size, age, gender and type of the cardiac surgery; (III) interventions and control; (IV) reported outcomes.

The same two investigators independently assessed the bias of the studies according to the Cochrane Collaboration's Risk of Bias. We assessed included random sequences generation, allocation concealment, blinding of participants and personnel, blinding of outcome assessment, incomplete outcome data, selective outcome reporting, and other sources of bias. Assessed criterion included low risk of bias, high risk of bias, and unclear. All disagreements were resolved by a third investigator.

\section{Statistical analysis}

Statistical analysis was undertaken by using Cochrane systematic review software Review Manager (RevMan; Version 5.3.5). For dichotomous outcomes, risk rate (RR) with $95 \%$ confidence intervals (CIs) were used to express the difference, and for continuous outcomes, mean difference (MD) or standardized mean difference (SMD) with $95 \%$ CIs were used to express the difference. MD was used to analysis the outcomes measured by the same method and the same unit, otherwise, SMD was used. $\mathrm{I}^{2}$ statistic was used to assess the heterogeneity. Studies with $\mathrm{I}^{2}<25 \%$ were considered to have low heterogeneity, $\mathrm{I}^{2}$ of $25-75 \%$ indicates medium heterogeneity and $\mathrm{I}^{2}>75 \%$ implies high heterogeneity. If $\mathrm{I}^{2}$ was $>50 \%$, we used the random effect model (RE) to combine the effect sizes, and if $\mathrm{I}^{2}$ was $<50 \%$, fixed effect model (FE) was used. Potential heterogeneity sources were identified by subgroup analyses or sensitivity analyses. Sensitivity analyses were conducted by omitting one study in each turn and investigating the influence of a single study on the overall pooled estimate. A P value $<0.05$ for all outcome measures was considered as statistically significant. The results were displayed as Forest plots.

\section{Results}

\section{Results of the search}

We retrieved 1,120 relevant articles through the computerized search (PubMed 457 articles, Web of Science 166 articles, Cochrane Central 118 articles, Embase 379 articles). After 299 duplicate studies were identified, we removed 807 articles by reading titles and abstracts.

Five articles were excluded after full-article reading as shown in the Figure 1. Finally, nine articles were included in this review and eight articles were included in the metaanalysis for the study performed by Araújo-Filho in 2017 
failed to provided data for quantitative meta-analysis (Figure 1).

\section{Characteristics of the included trials}

The nine studies met the inclusion criteria involving 830 participants (NIV vs. control: 449 vs. 381) who underwent cardiac surgery. Among the studies included, six studies applied CPAP for NIV, and four studies applied BiPAP. Seven studies compared NIV plus standard therapy with standard therapy alone. This comparison allowed us to determine the effects of using NIV as an adjunct to standard therapy. On the other hand, two studies aimed to compare the effectiveness of NIV with IS by setting NIV plus standard therapy other than IS as intervention groups while setting IS plus other standard therapy as control groups.

Standard therapy included the combination of the following treatment: oxygen therapy, CP, nebulized bronchodilators, coughing exercises, nebulized saline, mobilization and IS (10). The main characteristics of the included trials are presented in Table 1.

\section{Risk of bias in included studies}

Two investigators assessed the quality of the included studies independently based on the recommended criteria of the Cochrane Handbook. See Figures 2,3 and Table 2 for details.

\section{Effects of NIV and pulmonary complications}

NIV failed to reduce the incidence of atelectasis of post cardiac surgery patients

Four studies described quantitative measures of atelectasis included 278 participants (NIV vs. control: 157 vs. 121) (14-17). Meta-analysis of these studies showed a high statistical heterogeneity $\left(\mathrm{I}^{2}=69 \%\right)$, and NIV failed to reduce the incidence of atelectasis significantly (RR 0.60; 95\% CI: 0.28 to $1.28, \mathrm{P}=0.19$ ) in total.

Subgroup analysis of control groups showed no statistical heterogeneity $\left(\mathrm{I}^{2}=0 \%\right)$ and NIV still failed to exert any improvement (NIV + standard therapy vs. standard therapy: RR 0.60 ; $95 \%$ CI: 0.21 to $1.78, \mathrm{P}=0.36$ or NIV vs. IS: RR 0.51; $95 \%$ CI: 0.23 to $1.11, \mathrm{P}=0.09$ ) (Figure 4). Subgroup analysis of ventilation patterns also found no significant improvement or heterogeneity between subgroups (BiPAP: RR 0.37 ; $95 \%$ CI: 0.04 to $3.34, \mathrm{P}=0.38$ or CPAP: RR 1.14; $95 \%$ CI: 0.56 to $2.35, \mathrm{P}=0.72$ ) (Figure 5). However, the heterogeneity among the rest four studies decreased to $\mathrm{I}^{2}=16 \%$ without any significant difference between NIV group and control group after Al Jaaly 2013 was taken out from the analysis, which showed a more favorable result for NIV.

\section{NIV failed to reduce the incidence of pneumonia of post cardiac surgery patients}

Three studies used NIV as an adjunct to standard therapy reported this outcome, included 448 (NIV vs. control: 224 vs. 224) participants $(14,15,18)$. We combined them in a subgroup analysis by BiPAP (included 126 participants, NIV vs. control: 63 vs. 63) and CPAP (included 322 participants, NIV vs. control: 161 vs. 161) ventilation mode.

Meta-analysis of these studies showed no statistical heterogeneity $\left(\mathrm{I}^{2}=0 \%\right)$, and NIV failed to reduce the incidence of pneumonia (RR 0.27; 95\% CI: 0.05 to 1.64 , $\mathrm{P}=0.16$ ) in total (Figure 6).

The analysis showed no statistical heterogeneity $\left(\mathrm{I}^{2}=0 \%\right)$ between subgroups, and still we found NIV exert no significant improvement in both subgroup analysis (BiPAP: RR 0.20; $95 \%$ CI: 0.01 to $4.08, \mathrm{P}=0.30$ or CPAP: RR 0.33; 95\% CI: 0.04 to $3.17, \mathrm{P}=0.34$ ) as well.

\section{NIV shortened the length of ICU stay of post cardiac surgery patients}

There were five studies reported the measurement of the length of hospital stay (14,17-20). However, since two studies presented insufficient data, we only included three studies in this analysis, including 446 participants (NIV vs. control: $244 v s .202)(17,18,20)$. Two of the included studies compared CPAP plus standard therapy with standard therapy alone ( 350 participants included NIV vs. control: $178 v s .172)(18,20)$, and the other one compared NIV with IS (96 participants included, CPAP vs. BiPAP vs. control: 33 vs. 33 vs. 30) (17).

The meta-analysis included these three studies showed that there is no statistical heterogeneity $\left(\mathrm{I}^{2}=0 \%\right)$ between them, and indicated that the prophylactic use of NIV post cardiac surgery may led to a decrease in the length of ICU stay (MD -1.00 h, $95 \%$ CI: -1.38 to $-0.63, \mathrm{P}<0.00001$ ) (Figure 7).

A subgroup analysis of control group showed that there was no statistical heterogeneity between subgroups $\left(\mathrm{I}^{2}=0 \%\right)$, and we found that the use of NIV led to a decrease in the length of ICU stay in the subgroup which compared NIV plus standard therapy with standard therapy alone (MD -1.00 h, $95 \%$ CI: -1.38 to $-0.62, \mathrm{P}<0.00001)$, while no 


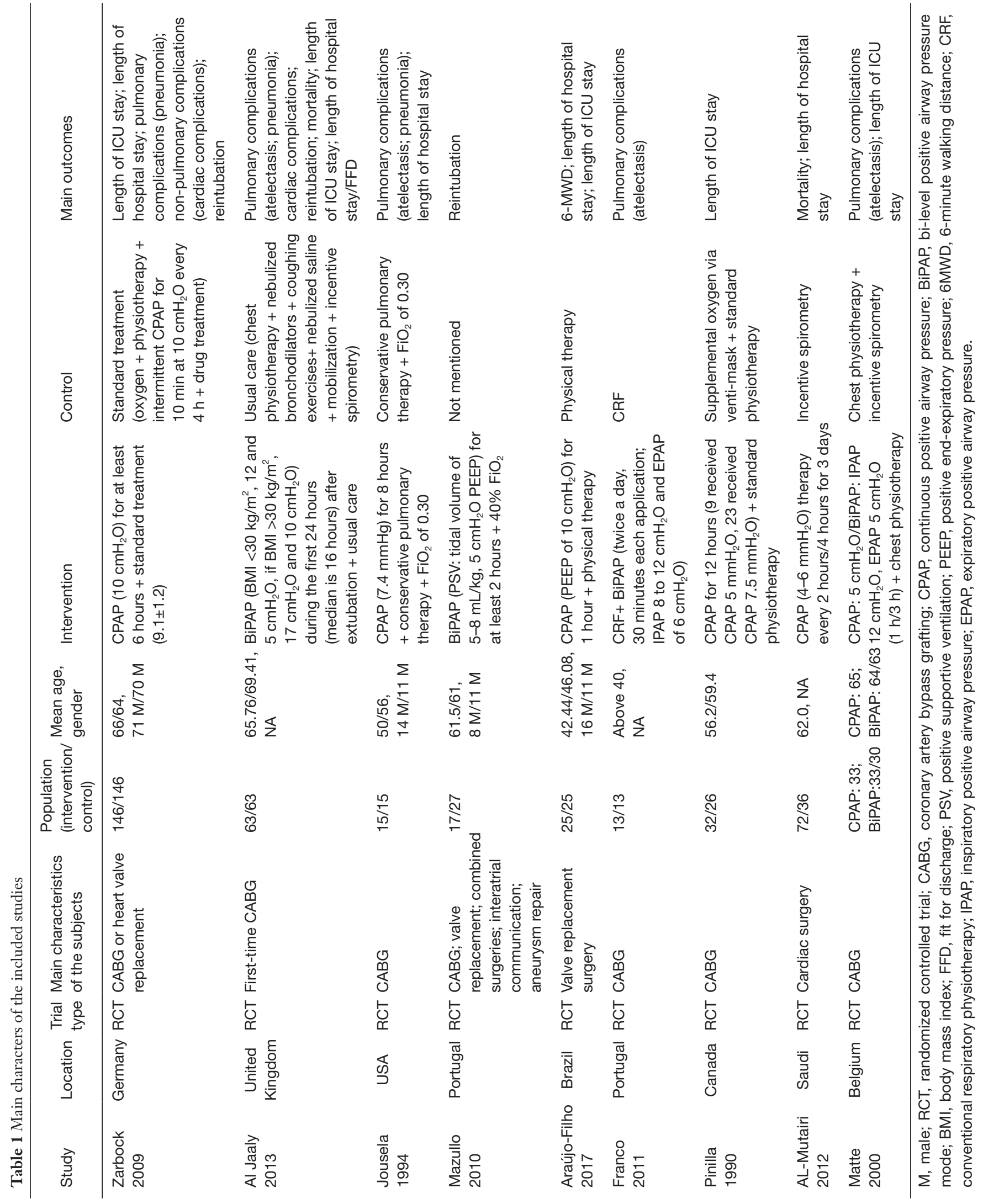




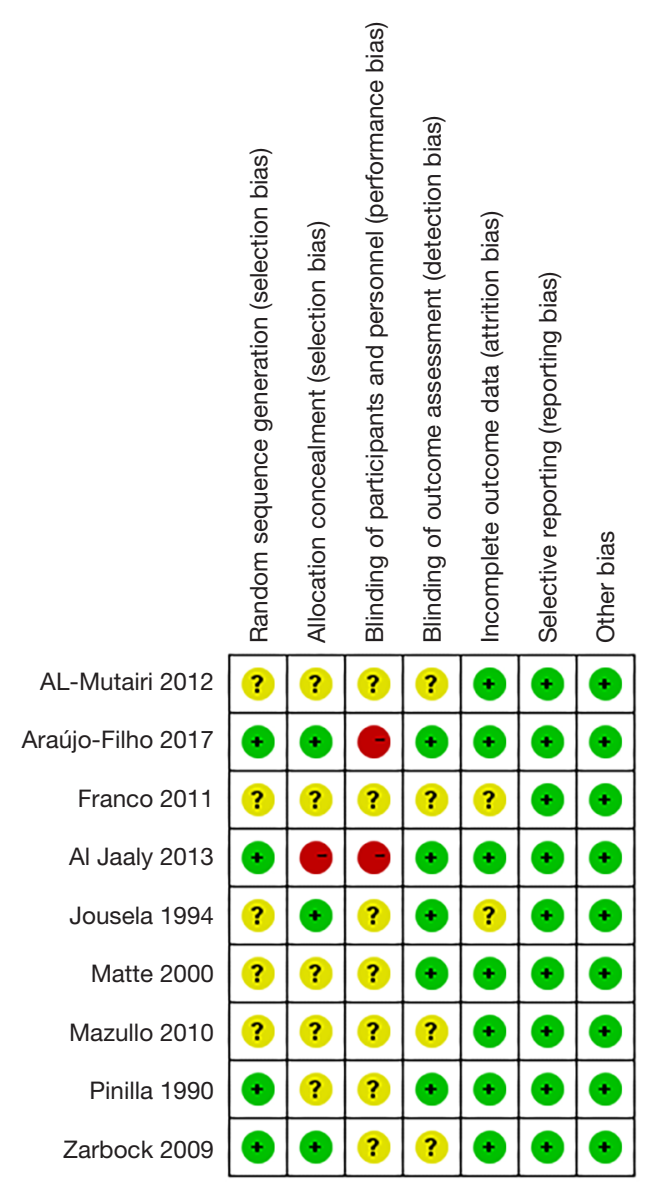

Figure 2 Methodological quality summary graph. difference was found in the subgroup which compared NIV with IS (MD -3.20 h, $95 \%$ CI: -10.92 to $4.53, \mathrm{P}=0.42$ ).

The other two studies were not included in the metaanalysis due to lack of data. They compared NIV plus standard therapy with standard therapy alone, and found NIV failed to exert any difference in the length of hospital stay (Al Jaaly 2013: RR 1.20, 95\% CI: 0.84 to 1.71; AraújoFilho 2017: $\mathrm{P}=0.55)(14,19)$.

\section{NIV shortened the length of hospital stay of post cardiac surgery patients}

Five studies reported the measurement of the length of hospital stay $(14,15,18,19,21)$. However, since three studies failed to present sufficient data for the analysis, we only include two studies in this analysis, including 322 participants (NIV vs. control: 161 vs. 161) $(15,18)$. Both of included studies compared CPAP plus standard therapy with standard therapy alone.

The meta-analysis showed a low statistic heterogeneity between the two included studies $\left(\mathrm{I}^{2}=13 \%\right)$, and indicated that the use of NIV may led to a decrease in the length of hospital stay (MD $-1.00 \mathrm{~d}, 95 \% \mathrm{CI}:-1.12$ to -0.87 , $\mathrm{P}<0.00001$ ) (Figure 8).

Among the other three studies which were not included in meta-analysis due to lack of data, two studies compared NIV plus standard therapy with standard therapy alone stated that the use of NIV failed to shorten the length of

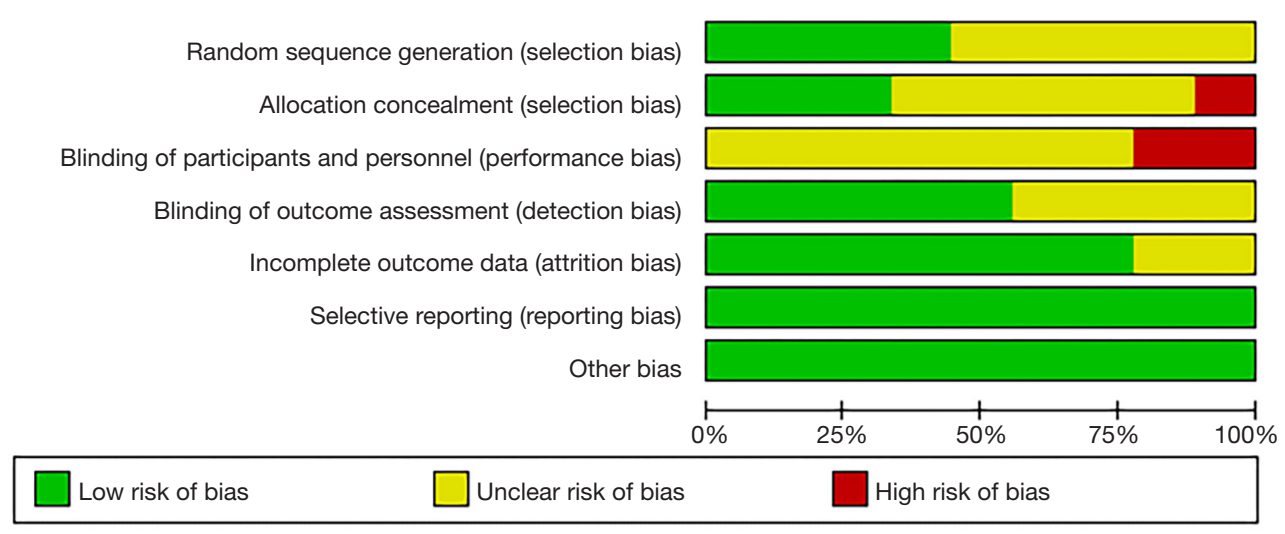

Figure 3 Methodological quality graph. 
Table 2 Risk of bias for included studies

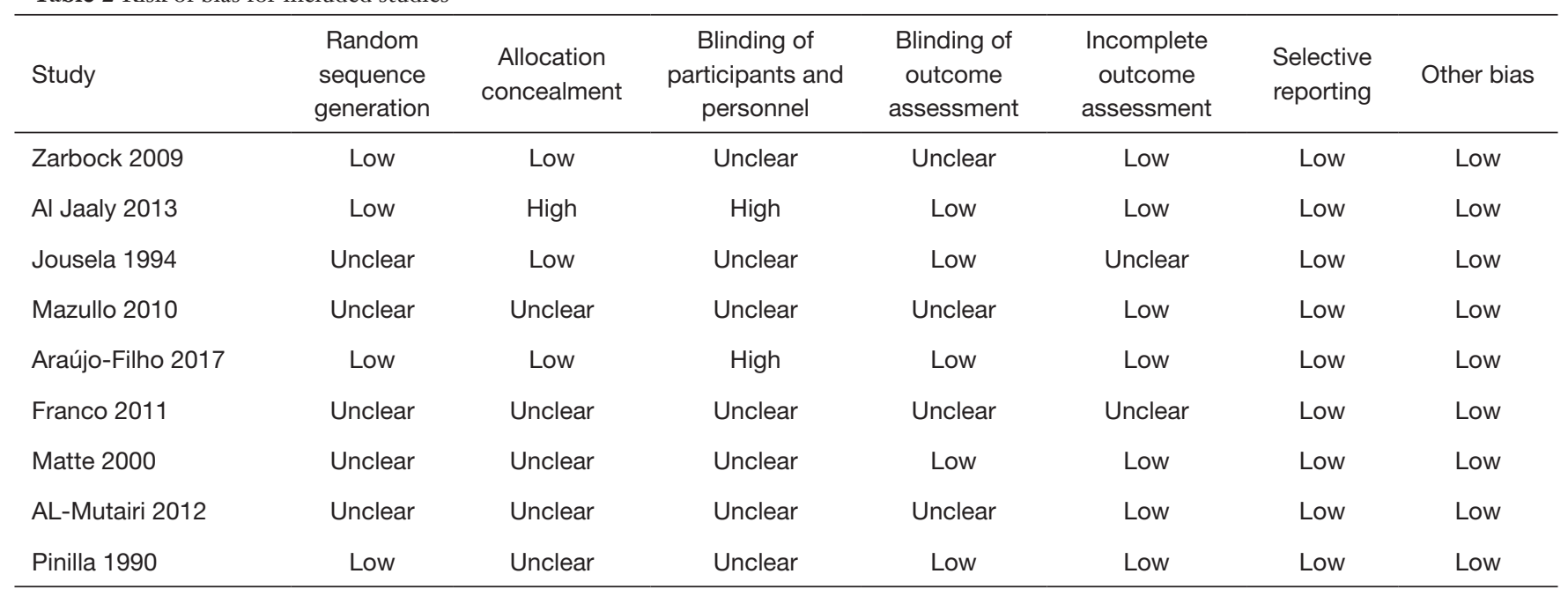

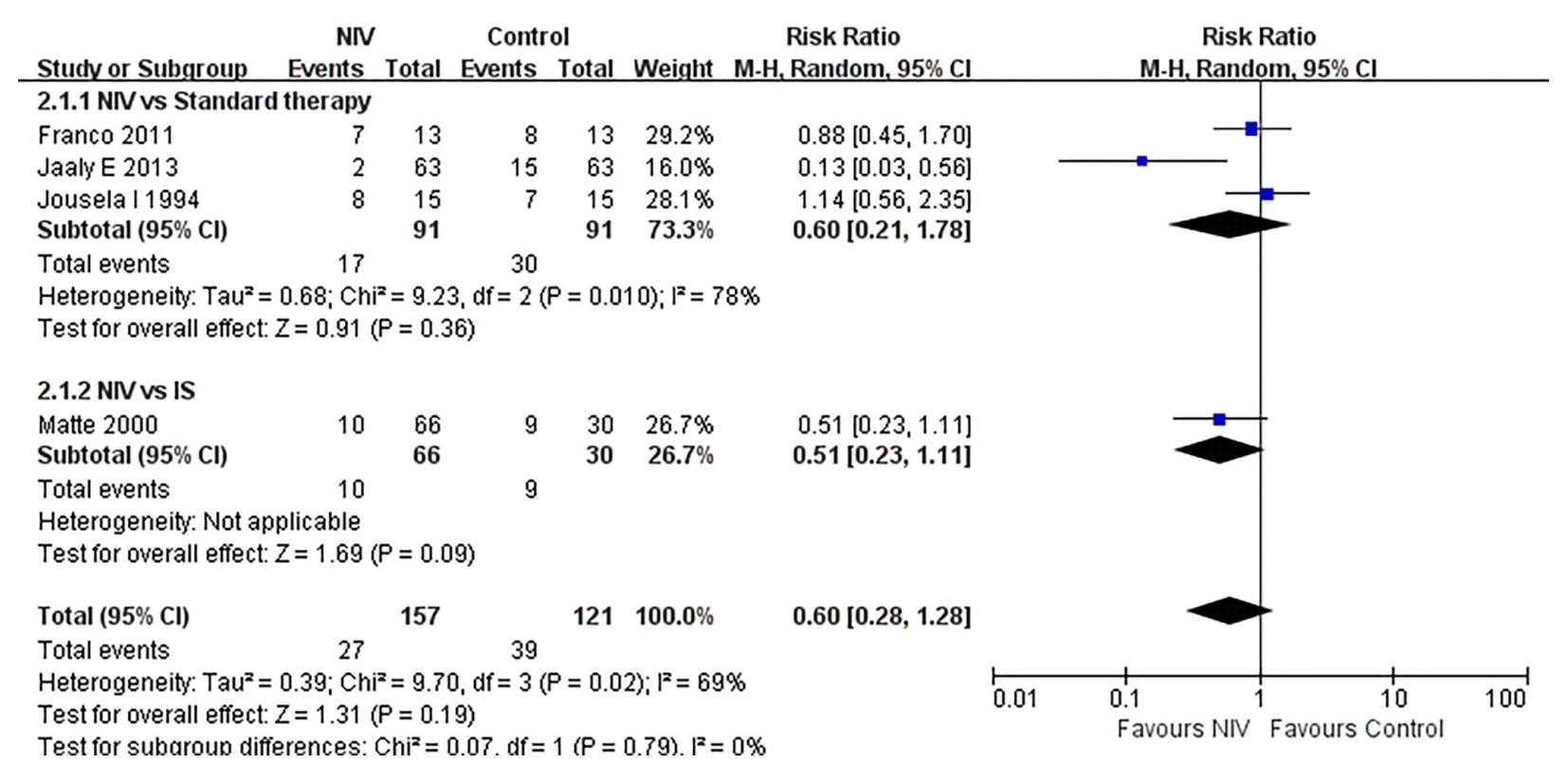

Figure 4 Forest plot of comparison: NIV vs. no NIV, outcome: atelectasis. NIV, non-invasive ventilation; IS, incentive spirometry.

hospital stay (Al Jaaly 2013: RR 1.12, 95\% CI: 0.78 to 1.60 ; Araújo-Filho 2017: $\mathrm{P}=0.41$ ). Al-Mutairi 2012 aimed to compare CPAP with IS failed to provide enough evidence for this outcome.

\section{NIV failed to reduce the rate of reintubation of post cardiac surgery patients}

Three studies compared NIV plus standard therapy with standard therapy alone reported this outcome, included 462 (NIV vs. control: 226 vs. 236) participants $(14,18,22)$. We combined them in a meta-analysis by BiPAP (included 170 participants, NIV vs. control: $80 v s$. 90) and CPAP (included 292 participants, NIV vs. control: 146 vs. 146) ventilation mode.

Meta-analysis of these studies showed no statistical heterogeneity $\left(\mathrm{I}^{2}=0 \%\right)$, and NIV failed to reduce the rate of reintubation (RR 0.68 ; $95 \%$ CI: 0.21 to $2.26, \mathrm{P}=0.53$ ) in total.

The analysis showed a low statistical heterogeneity $\left(\mathrm{I}^{2}=23 \%\right)$ between subgroups divided by ventilation mode, 


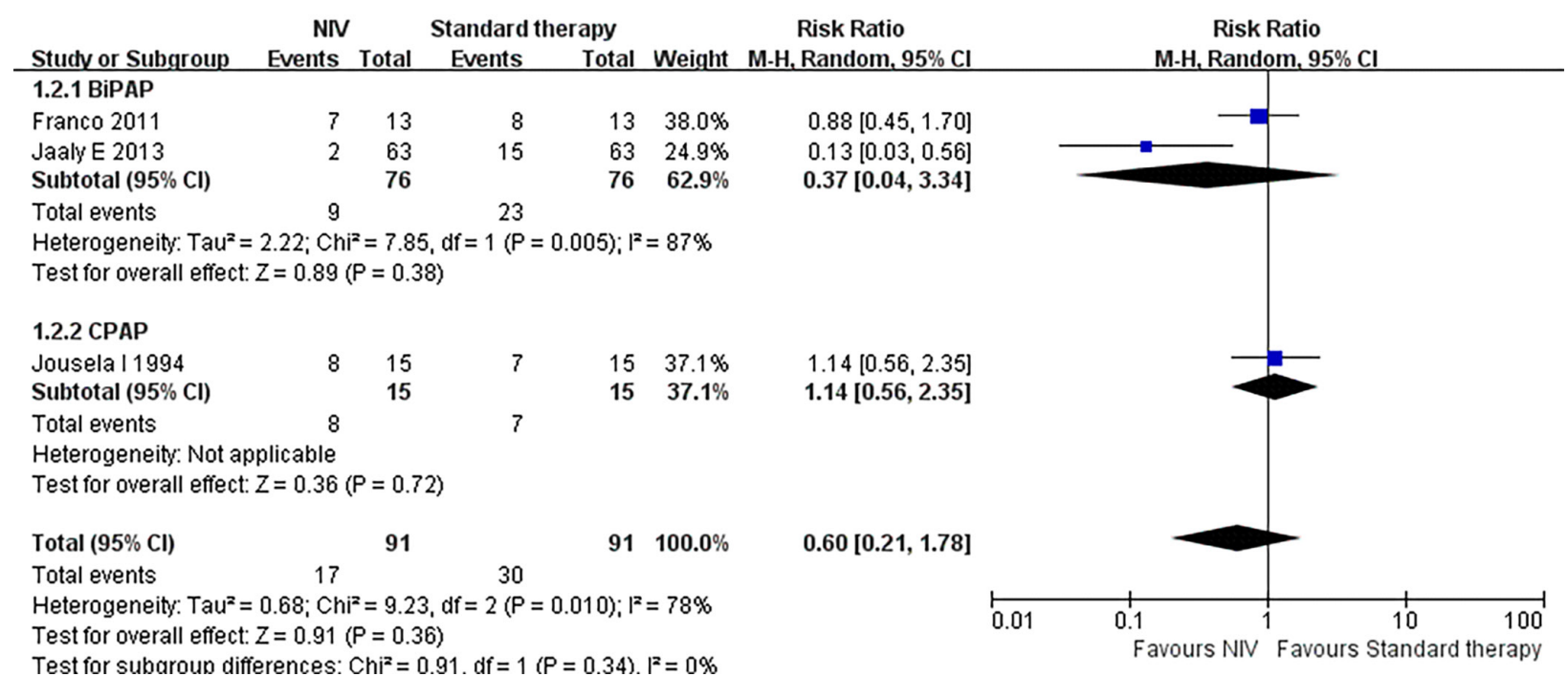

Figure 5 Forest plot of comparison: NIV plus standard therapy $v s$. standard therapy, outcome: atelectasis. NIV, non-invasive ventilation; BiPAP, bi-level positive airway pressure; CPAP, continuous positive airway pressure.

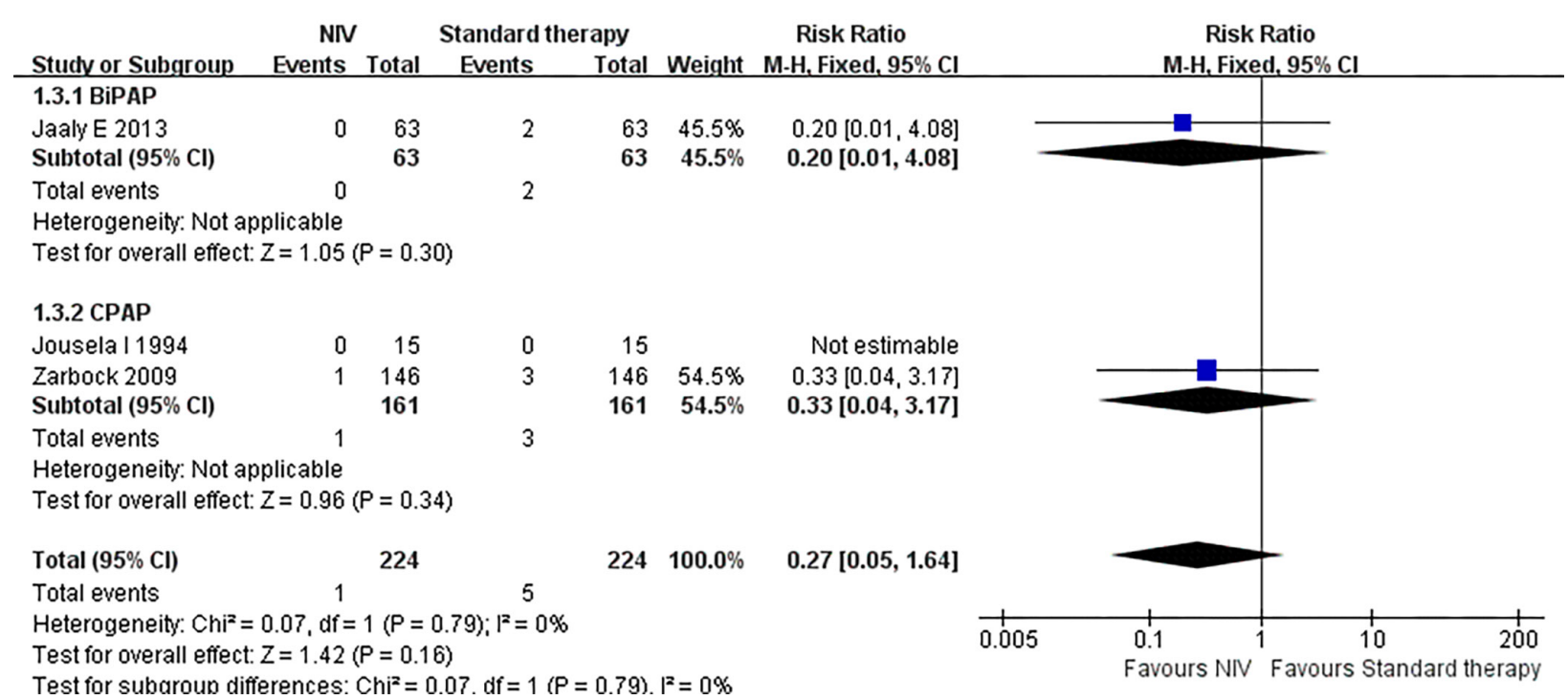

Figure 6 Forest plot of comparison: NIV plus standard therapy vs. standard therapy, outcome: pneumonia. NIV, non-invasive ventilation; BiPAP, bi-level positive airway pressure; CPAP, continuous positive airway pressure.

and subgroup analysis failed to show any improvement exert from NIV (BiPAP: RR 0.34; 95\% CI: 0.05 to 2.10, $\mathrm{P}=0.25$ or CPAP: RR 1.50 ; $95 \% \mathrm{CI}: 0.25$ to $8.85, \mathrm{P}=0.65)$ as well (Figure 9).

\section{NIV failed to reduce cardiac complications of post cardiac surgery patients}

Two studies reported this outcome with 418 participants
(NIV vs. control: 209 vs. 209) (14,18). Both of studies compared the use of NIV plus standard therapy with standard therapy alone. Al Jaaly 2013 applied BiPAP for ventilation mode, and Zarbock 2009 applied CPAP.

The meta-analysis showed no statistic heterogeneity between the two included studies $\left(\mathrm{I}^{2}=0 \%\right)$, and indicated that the prophylactic use of NIV post cardiac surgery failed to decrease the risk of cardiac complications (RR 0.81, 95\% 


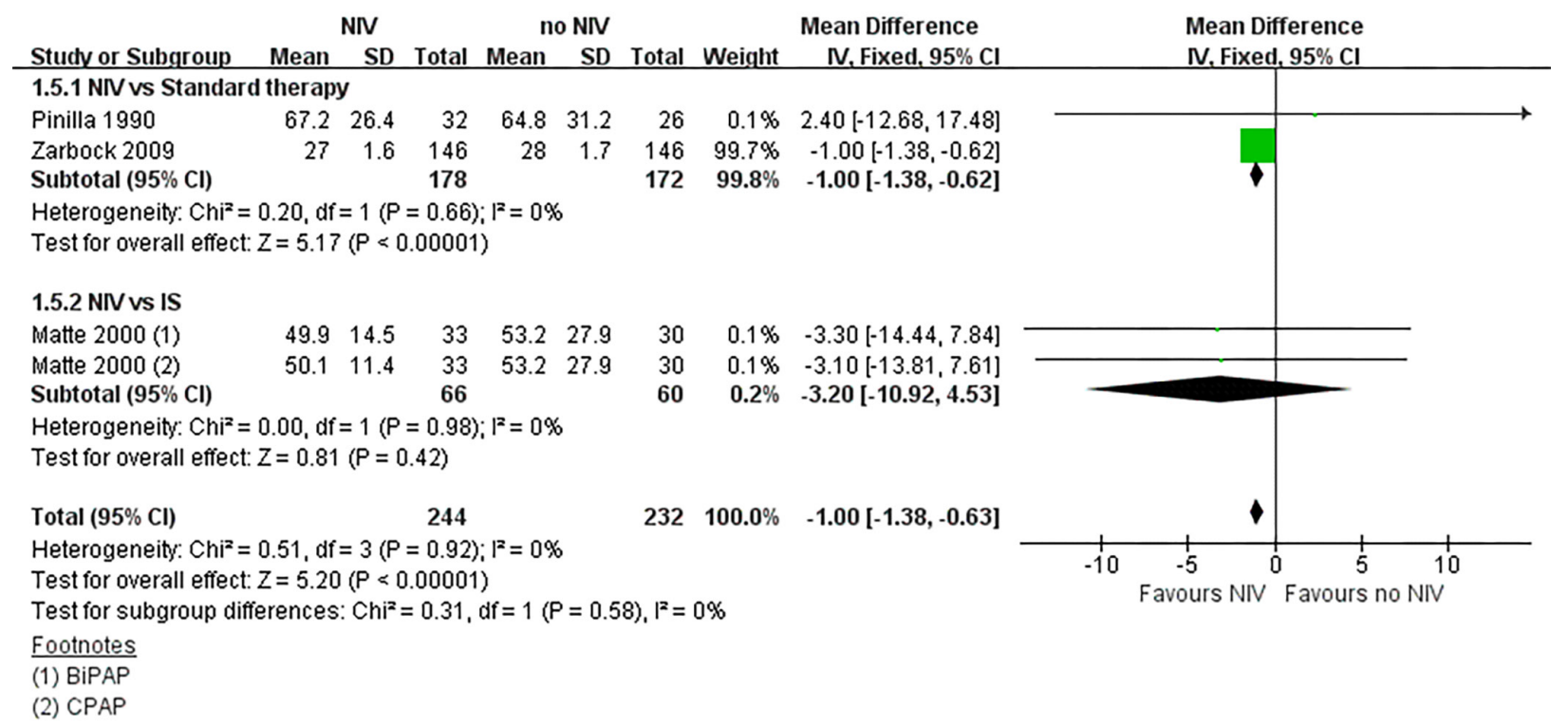

Figure 7 Forest plot of comparison: NIV vs. no NIV, outcome: length of ICU stay. NIV, non-invasive ventilation; IS, incentive spirometry; BiPAP, bi-level positive airway pressure; CPAP, continuous positive airway pressure.

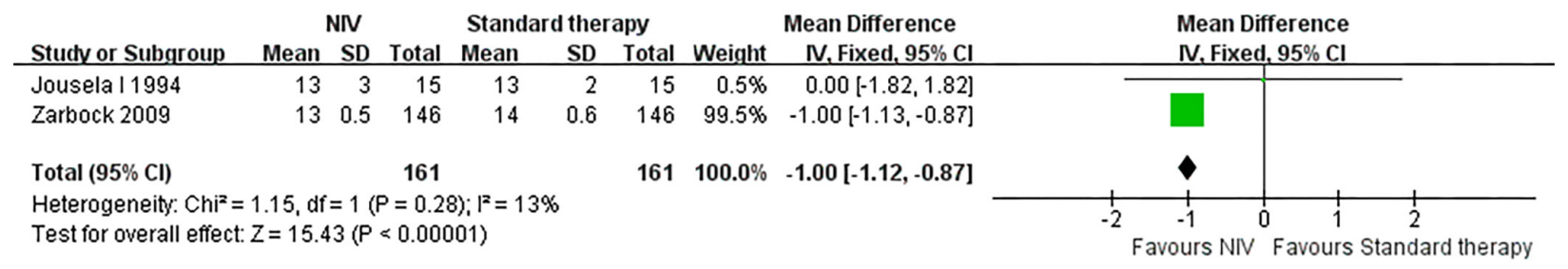

Figure 8 Forest plot of comparison: NIV plus standard therapy vs. standard therapy, outcome: length of hospital stay. NIV, non-invasive ventilation.

\section{CI: 0.59 to $1.13, \mathrm{P}=0.22$ ) (Figure 10 ).}

\section{NIV failed to reduce the mortality of post cardiac surgery patients}

Two studies reported the mortality of the subjects included 135 participants (NIV vs. control: 135 vs. 99), with one (Al Jaaly 2013) compared BiPAP plus standard therapy with standard therapy alone, and the other one (Al-Mutairi 2012) compared CPAP with IS. This analysis had no statistical heterogeneity $\left(\mathrm{I}^{2}=0 \%\right)(14,21)$.

Al Jaaly 2013, which included 126 participants (NIV vs. control: $63 v s .63)$, reported 1 patient died in NIV group while 1 died in control group (RR 1.00; $95 \% \mathrm{CI}: 0.06$ to 15.64).

Al-Mutairi 2012, which included 108 participants (NIV vs. control: 72 vs. 36), reported 3 participants died in NIV group, while no participant died in control group (RR 3.55; 95\% CI: 0.19 to 66.89 ).

We found NIV exert no significant difference of mortality between the NIV group and the control group through the meta-analysis (RR 2.02; 95\% CI: 0.29 to 14.05 , $\mathrm{P}=0.48$ ) (Figure 11).

NIV increased 6-MWD of post cardiac surgery patients Only one study reported the measurement of 6-MWD among the included studies (19), which compared the use of CPAP plus standard therapy with standard therapy alone with 50 participants (CPAP vs. control: $25 v s$. 25). The study showed the prophylactic use of NIV post cardiac surgery led to a significantly increase in 6-MWD (MD $69.73 \mathrm{~m}$, 


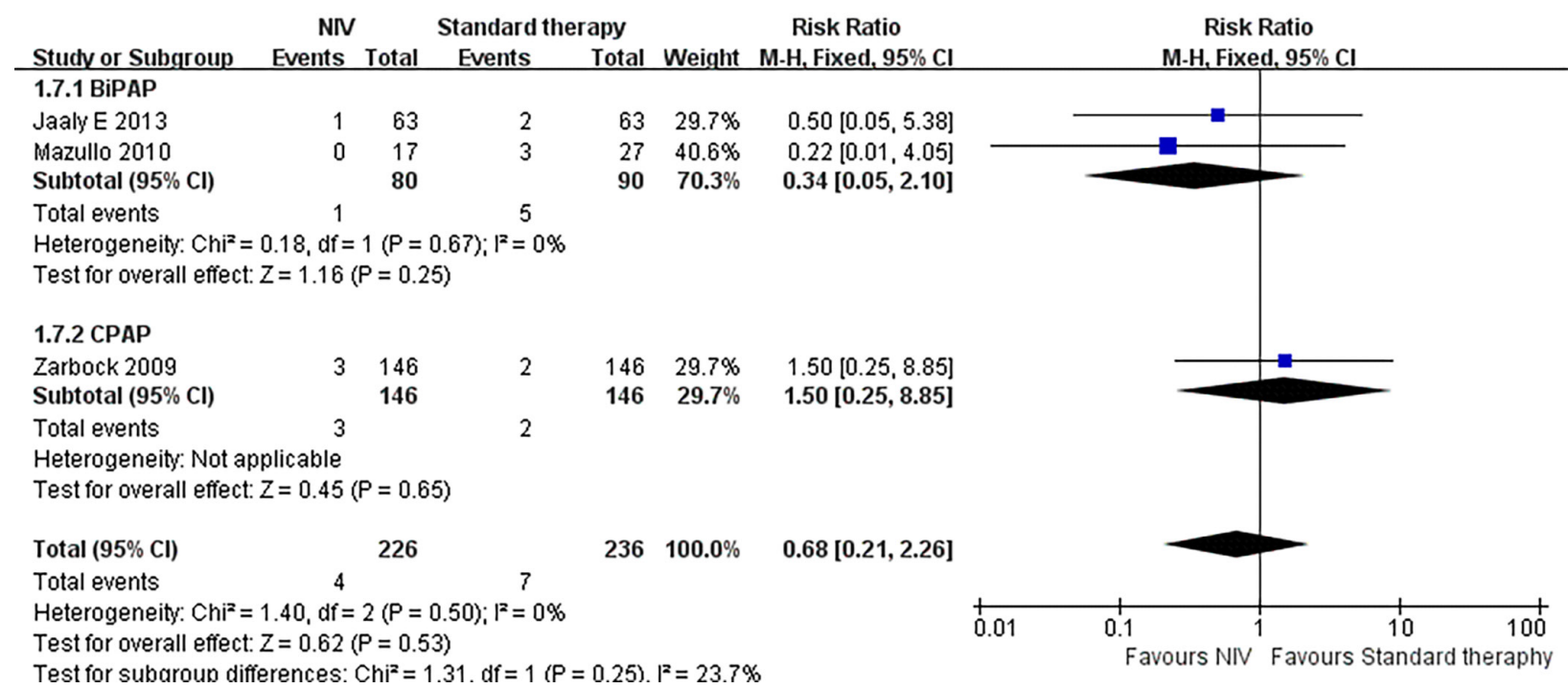

Figure 9 Forest plot of comparison: NIV plus standard therapy $v s$. standard therapy, outcome: rate of reintubation. NIV, non-invasive ventilation; BiPAP, bi-level positive airway pressure; CPAP, continuous positive airway pressure.

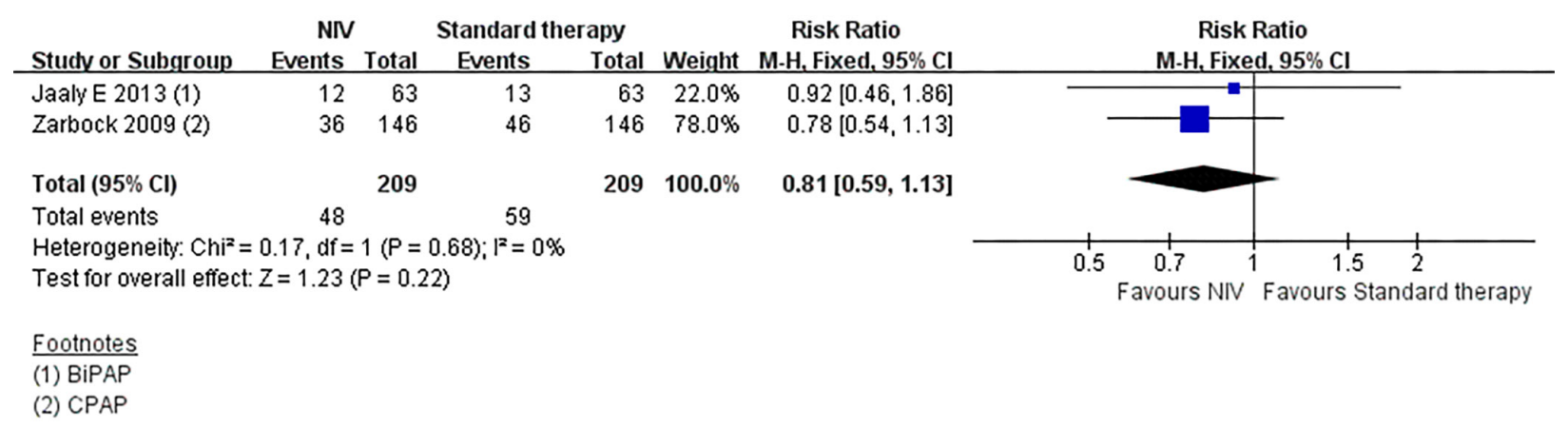

Figure 10 Forest plot of comparison: NIV plus standard therapy vs. standard therapy, outcome: cardiac complications. NIV, non-invasive ventilation; BiPAP, bi-level positive airway pressure; CPAP, continuous positive airway pressure.

\begin{tabular}{|c|c|c|c|c|c|c|c|c|c|c|}
\hline Study or Subgroup & $\begin{array}{c}\text { NIV } \\
\text { Events }\end{array}$ & Total & $\begin{array}{l}\text { Contrc } \\
\text { Events }\end{array}$ & $\begin{array}{l}\text { ol } \\
\text { Total }\end{array}$ & Weight & $\begin{array}{c}\text { Risk Ratio } \\
\text { M-H, Fixed, 95\% Cl }\end{array}$ & & $\begin{array}{r}\text { Risk } \\
\text { M-H, Fix } \\
\end{array}$ & $\begin{array}{l}\text { k Ratio } \\
\text { ked, } 95 \% \mathrm{Cl}\end{array}$ & \\
\hline ALMutairi 2012 (1) & 3 & 72 & 0 & 36 & $39.9 \%$ & $3.55[0.19,66.89]$ & & & $\square$ & - \\
\hline Jaaly E 2013 (2) & 1 & 63 & 1 & 63 & $60.1 \%$ & $1.00[0.06,15.64]$ & & & & \\
\hline Total $(95 \% \mathrm{Cl})$ & & 135 & & 99 & $100.0 \%$ & $2.02[0.29,14.05]$ & & & & \\
\hline Total events & 4 & & 1 & & & & & & & \\
\hline $\begin{array}{l}\text { Heterogeneity: } \text { Chi }^{2}= \\
\text { Test for overall effect }\end{array}$ & $\begin{array}{l}0.39, \mathrm{df}= \\
\mathrm{Z}=0.71(\mathrm{f}\end{array}$ & $\begin{array}{l}1(P= \\
P=0.4\end{array}$ & $\begin{array}{l}0.53) ; 1^{2}= \\
48)\end{array}$ & $0 \%$ & & & 0.01 & $\begin{array}{l}0.1 \\
\text { Favours NIV }\end{array}$ & ${ }_{1}^{1} \quad 10$ & 100 \\
\hline
\end{tabular}

Figure 11 Forest plot of comparison: NIV vs. no NIV, outcome: mortality. NIV, non-invasive ventilation; IS, incentive spirometry; BiPAP, bi-level positive airway pressure; CPAP, continuous positive airway pressure. 
95\% CI: 28.96 to $110.50, \mathrm{P}<0.001)$.

\section{Discussion}

The target of this study is to elaborate the effectiveness of the prophylactic use of NIV in subjects who underwent cardiac surgery. Post-operate pulmonary complications occurred frequently in patients went through cardiac surgery, with atelectasis be the most common one. Those complications usually led to an increase in hospital length of stay, morbidity, mortality, and cost for the health care system (2-4). Therefore, strategies, such as NIV, are in need to reduce the complications after the cardiac surgery.

The primary outcomes are pulmonary complications (including atelectasis and pneumonia), the length of ICU stay and the length of hospital stay.

We assumed the reasons for the failure of NIV to reduce pulmonary complications in several studies may be the following. First, since atelectasis was more common in patients with internal thoracic artery grafting and pleural drainage (15), the difference of the ratio of their presence in intervention groups and control groups (which is not reported in every study included) may exert influence on outcomes. Phrenic nerve injury during surgery are often seen, and it would be expected to cause atelectasis and other complications soon after the removal of mechanical ventilation, therefore the use of NIV only delay the occur of atelectasis, but failed to prevent it from happening (15). Moreover, decreased mobility in bed, pain after surgery and lung damage during surgery are risk factors contribute to post surgery complications $(23,24)$, which couldn't be altered by NIV (16). However, when we performed the analysis on the incident of atelectasis, we noticed the high heterogeneity among studies was brought out by $\mathrm{Al}$ Jaaly 2013 which showed a more favorable result for NIV. After assessed the four studies reported this outcome, we believed the length of the NIV time were contributed to the heterogeneity. Al Jaaly 2013 applied NIV during the first 24 hours (median is 16 hours), while the other three studies are 8 hours 30 minutes each application (1 hour in total) and every 1 hour for 3 hours ( 8 hours in total) (14-17). This outcome might indicate that we may be able to decrease the risk of post-cardiac surgery atelectasis with longer NIV time, but more evidence and RCTs are needed in the future to prove the assumption.

The length of hospital stay and ICU stay in our study show discrepancy compared with those in a previous study by Pieczkoski 2017 (25). Although Pieczkoski et al. included the same three RCTs and determined the length of ICU stay, they extracted the data from Zarbock 2009 incorrectly, which led to an opposite conclusion. As for the length of hospital stay, they failed to conduct a meta-analysis due to the lack of RCTs.

Since NIV failed to reduce pulmonary complications, we assumed it shortened the length of ICU and hospital stay through other mechanisms. After reading through studies included in the analyses of the length of hospital stay and ICU stay, we found that Zarbock 2009 reported NIV significantly decreased the occurrence of hypoxemia event $\left(\mathrm{PaO}_{2} / \mathrm{FiO}_{2}<100\right)$. Jousela 1994 and Pinilla 1990 reported the decrease of $\mathrm{PaO}_{2} / \mathrm{FiO}_{2}$ after surgery significantly reduced in NIV treatment group. Matte 2000 reported NIV significantly lightened the reduce of FEV1. For these reasons, we assumed the length of hospital stay and ICU stay might be shortened by the increase of $\mathrm{PaO}_{2} / \mathrm{FiO}_{2}$ and the improvement of pulmonary function in these studies. However, this hypothesis still needs to be confirmed by follow-up researches. In addition, since two studies excluded from the analyses of the length of hospital stay and ICU stay (Al Jaaly 2013 and Araújo-Filho 2017) due to lack of data failed to reported any improvement through NIV treatment, the benefit of NIV treatment in these two outcomes still in need of more RCTs to provide supportive evidence.

The secondary outcomes are rate of reintubation, cardiac complications, mortality and exercise capacity (6MWD). Our study failed to find any significant change in the rate of reintubation, mortality and cardiac pulmonary complications.

Although we failed to find any significant difference in rate of reintubation when NIV was applied as an adjunct to standard therapy, we did observe a tendency to reduce reintubation in the subgroup applied BiPAP. We failed to find the same tendency in the subgroup applied CPAP. A moderate heterogeneity was found between subgroups, which suggested that the mode of ventilation may have an impact on the outcomes, but more trials are expected to be carried out in the future.

Functional capacity is limited in patients who went through cardiac surgery due to many factors, including abnormal ventilatory responses, peripheral muscle dysfunction and cardiac dysfunction $(20,26)$. The 6-minute walk test (6MWT) is a useful evaluation tool for exercise capacity because the test is simple and cost-effective (27). Only one study reported this outcome with a significant improvement, therefore, more RCTs are expected in the 
future.

Compared with the previous meta-analysis, out study have the following improvement. First, we included a new RCT published in 2017 that reported the effectiveness of applying NIV immediately after cardiac surgery. Second, we divided our studies into subgroups according to ventilation mode (BiPAP and CPAP) and their control groups, in order to figure out whether the type of modes have an impact on the outcomes and eliminate the bias cause by different control groups. Third, we excluded one article from the previous meta-analysis which aimed to applied NIV on early extubation subjects. We decided the timing of extubation would have an impact on the outcomes. Forth, we added cardiac complications and exercise capacity (6-MWD) as outcomes of our study.

However, our study is limited by the small sample size. The methodological quality of the RCTs included were unsatisfying, and it was almost inevitable for most of them failed to report allocation concealment and blindness properly due to the process of NIV treatment. Difference in the parameters and length of ventilation applied in NIV groups may increase the bias of our outcomes as well. In order to determine the effectiveness of applying NIV in subjects went through cardiac surgeries, more RCTs with better methodological methods and larger sample size are expected to be presented in the future, and we expect to find more RCTs applying NIV with longer ventilation time to figure out whether the length of NIV time have an impact on the outcomes.

\section{Conclusions}

There was no significant difference in pulmonary complications between prophylactic NIV and standard therapy in post-cardiac surgery subjects. However, it indicated that the incidence of pulmonary complications of post-cardiac surgery subjects may be reduced by prolonged NIV treatment. Our study showed that the application of NIV may shorten the length of hospital stay and the length of ICU stay, but there was no significant difference between the prophylactic NIV and standard therapy in mortality, rate of reintubation and cardiac complications. Therefore, after careful consideration, we recommend the use of NIV after cardiac surgery to shorten the length of hospital stay and ICU stay, and the use of NIV with prolonged treatment to prevent pulmonary complications. However, due to the low methodological quality and the small sample size of the included study, more RCTs with high quality are expected in the future to expand the information.

\section{Acknowledgments}

Funding: This work was supported by grants from The National Key Research and Development Program of China (No. 2018YFC1313600) and the National Natural Science Foundation of China (No. 81570081, 81770083).

\section{Footnote}

Conflicts of Interest: All authors have completed the ICMJE uniform disclosure form (available at http://dx.doi. org/10.21037/jtd.2020.02.30). The authors have no conflicts of interest to declare.

Ethical Statement: The authors are accountable for all aspects of the work in ensuring that questions related to the accuracy or integrity of any part of the work are appropriately investigated and resolved.

Open Access Statement: This is an Open Access article distributed in accordance with the Creative Commons Attribution-NonCommercial-NoDerivs 4.0 International License (CC BY-NC-ND 4.0), which permits the noncommercial replication and distribution of the article with the strict proviso that no changes or edits are made and the original work is properly cited (including links to both the formal publication through the relevant DOI and the license). See: https://creativecommons.org/licenses/by-nc-nd/4.0/.

\section{References}

1. Weiser TG, Haynes AB, Molina G, et al. Estimate of the global volume of surgery in 2012: an assessment supporting improved health outcomes. Lancet 2015;385 Suppl 2:S11.

2. Chiumello D, Chevallard G, Gregoretti C. Non-invasive ventilation in postoperative patients: a systematic review. Intensive Care Medicine 2011;37:918-29.

3. Warner DO. Preventing postoperative pulmonary complications: the role of the anesthesiologist. Anesthesiology 2000;92:1467.

4. Gardner B, Palasti S. A comparison of hospital costs and morbidity between octogenarians and other patients undergoing general surgical operations. Surg Gynecol Obstet 1990;171:299-304.

5. Niyayeh Saffari NH, Nasiri E, Mousavinasab SN, et 
al. Frequency Rate of Atelectasis in Patients Following Coronary Artery Bypass Graft and Its Associated Factors at Mazandaran Heart Center in 2013-2014. Glob J Health Sci 2015;7:97-105.

6. Landymore RW, Howell F. Pulmonary complications following myocardial revascularization with the internal mammary artery graft. Eur J Cardiothorac Surg 1990;4:156-61.

7. Lopes CR, Brandão CM, Nozawa E, et al. Benefits of noninvasive ventilation after extubation in the postoperative period of heart surgery. Rev Bras Cir Cardiovasc 2008;23:344-50.

8. Stock MC, Downs JB, Gauer PK, et al. Prevention of Postoperative Pulmonary Complications with CPAP, Incentive Spirometry, and Conservative Therapy. Chest 1985;87:151-7.

9. Mehta S. Noninvasive positive pressure ventilation in acute respiratory failure. Intensive Care Med 1998;24:1113-4.

10. Torres MF, Porfirio GJ, Carvalho AP, et al. Noninvasive positive pressure ventilation for prevention of complications after pulmonary resection in lung cancer patients. Cochrane Database Syst Rev 2015;(9):CD010355.

11. Duncan SR, Negrin RS, Mihm FG, et al. Nasal continuous positive airway pressure in atelectasis. Chest 1987;92:621-4.

12. Kindgen-Milles D, Buhl R, Loer SA, et al. Nasal CPAP therapy: effects of different CPAP levels on pressure transmission into the trachea and pulmonary oxygen transfer. Acta Anaesthesiol Scand 2002;46:860-5.

13. Lenique F, Habis M, Lofaso F, et al. Ventilatory and hemodynamic effects of continuous positive airway pressure in left heart failure. Am J Respir Crit Care Med 1997;155:500-5.

14. Al Jaaly E, Fiorentino F, Reeves BC, et al. Effect of adding postoperative noninvasive ventilation to usual care to prevent pulmonary complications in patients undergoing coronary artery bypass grafting: a randomized controlled trial. J Thorac Cardiovasc Surg 2013;146:912-8.

15. Jousela I, Räsänen J, Verkkala K, et al. Continuous positive airway pressure by mask in patients after coronary surgery. Acta Anaesthesiologica Scandinavica 1994;38:311-6.

16. Franco AM, Torres FC, Simon IS, et al. Assessment of noninvasive ventilation with two levels of positive airway pressure in patients after cardiac surgery. Rev Bras Cir Cardiovasc 2011;26:582-90.

17. Matte P, Jacquet L, Van DM, et al. Effects of conventional physiotherapy, continuous positive airway pressure and non-invasive ventilatory support with bilevel positive airway pressure after coronary artery bypass grafting. Acta
Anaesthesiologica Scandinavica 2000;44:75-81.

18. Zarbock A, Mueller ES, Netzer S, et al. Prophylactic nasal continuous positive airway pressure following cardiac surgery protects from postoperative pulmonary complications: a prospective, randomized, controlled trial in 500 patients. Chest 2009;135:1252-9.

19. Araújo-Filho AA, Cerqueira-Neto ML, Cacau LA, et al. Effect of prophylactic non-invasive mechanical ventilation on functional capacity after heart valve replacement: a clinical trial. Clinics 2017;72:618-23.

20. Pinilla JC, Oleniuk FH, Tan L, et al. Use of a nasal continuous positive airway pressure mask in the treatment of postoperative atelectasis in aortocoronary bypass surgery. Crit Care Med 1990;18:836-40.

21. Al-Mutairi FH, Fallows SJ, Abukhudair WA, et al. Difference between continuous positive airway pressure via mask therapy and incentive spirometry to treat or prevent post-surgical atelectasis. Saudi Med J 2012;33:1190-5.

22. Mazullo Filho JB, Bonfim VJ, Aquim EE. Noninvasive mechanical ventilation in immediate postoperative cardiac surgery patients. Rev Bras Ter Intensiva 2010;22:363-8.

23. Carvalho ACC, Oliveira EM, Souza JAM. Pós-operatório em cirurgia cardíaca. In: Knobel E. editor. Condutas no paciente grave. 2nd edition. São Paulo: Atheneu, 1998:1031-42.

24. Taylor GJ, Mikell FL, Moses HW, et al. Determinants of hospital charges for coronary artery bypass surgery: the economic consequences of postoperative complications. Am J Cardiol 1990;65:309-13.

25. Pieczkoski SM, Margarites AGF, Sbruzzi G. Noninvasive Ventilation During Immediate Postoperative Period in Cardiac Surgery Patients: Systematic Review and MetaAnalysis. Braz J Cardiovasc Surg 2017;32:301-11.

26. Landoni G, Zangrillo A, Cabrini L. Noninvasive Ventilation After Cardiac and Thoracic Surgery in Adult Patients: A Review. J Cardiothorac Vasc Anesth 2012;26:917-22.

27. Guimarães GV, Carvalho VO, Bocchi EA. Reproducibility of the self-controlled six-minute walking test in heart failure patients. Clinics 2008;63:201-6.

Cite this article as: $\mathrm{Wu} \mathrm{Q}$, Xiang $\mathrm{G}$, Song $\mathrm{J}$, Xie L, Wu X, Hao S, Wu X, Liu Z, Li S. Effects of non-invasive ventilation in subjects undergoing cardiac surgery on length of hospital stay and cardiac-pulmonary complications: a systematic review and meta-analysis. J Thorac Dis 2020;12(4):1507-1519. doi: 10.21037/ jtd.2020.02.30 